\title{
Compare different spatial based fuzzy-C_mean (FCM) extensions for MRI image segmentation
}

\begin{abstract}
FCM does not use spatial information in clustering process. Therefore, it is not robust against noise and other imaging artefacts. In order to incorporate spatial information, an extension for FCM (FCM_S) is proposed which allows pixel to be labelled by influence of its neighbourhood labels. FCM_S is time-consuming. To over come this problem, FCM_S1 is introduced, which is faster. Then, FCM_EN and FGFCM are proposed which are faster than previous methods. Four spatial based extensions are simulated for FCM: FCM_S, FCM_S1, FCM_EN and FGFCM. In order to compare their quality, they are applied to simulated brain MRI images and similarity index is used to compare their quality quantitatively.
\end{abstract}

Keyword: Brain segmentation; FCM 